\title{
Quantitative estimation of lysosomal storage in mucopolysaccharidoses by electron microscopy analysis
}

\author{
Magdalena Narajczyk ${ }^{1 凶}$, Marta Moskot ${ }^{2}$ and Aleksandra Konieczna² \\ 'Laboratory of Electron Microscopy; ${ }^{2}$ Department of Molecular Biology, University of Gdańsk, Gdańsk, Poland
}

\begin{abstract}
Mucopolysaccharidoses (MPS) are severe inherited metabolic disorders caused by storage of glycosaminoglycans (GAGs). The level of accumulated GAGs is an important parameter in assessment of the severity of the disease and the efficacy of treatment; unfortunately, biochemical methods are often unreliable and only semi-quantitative. Therefore, finding other methods for estimation of GAG levels and/or assessment of the efficacy of applied therapy is very important. Although monitoring of GAG levels during therapy is crucial, in this work it is proposed that analysis of the ultrastructure of MPS cells by electron microscopic techniques can be considered as an alternative and reliable method for assessment of lysosomal storage. The number of complex lysosomal structures was found to be significantly higher in MPS cells relative to controls, while it decreased significantly in response to either enzyme replacement therapy or substrate reduction therapy. Thus, this parameter, easily assessed by electron microscopy, appears to correspond to the physiological state of MPS cells.
\end{abstract}

Key words: mucopolysaccharidoses, glycosaminoglycans, transmission electron microscopy

Received: 17 November, 2012; revised: 27 November, 2012 accepted: 06 December, 2012; available on-line: 11 December, 2012

\section{INTRODUCTION}

Mucopolysaccharidoses (MPS) are rare, inherited lysosomal storage disorders caused by deficiencies in activities of lysosomal hydrolases (Neufeld \& Muenzer, 2001). In these diseases, defects in degradation of glycosaminoglycans (GAGs) are caused by mutations in genes coding for these enzymes. This leads to accumulation of GAGs in lysosomes, which compromises the functioning of different tissues and organs and usually causes death in childhood (Neufeld \& Muenzer, 2001).

One of the most promising therapies of MPS is enzyme replacement therapy (ERT), which is now registered for three types of MPS (types I, II and VI). Unfortunately, this treatment is ineffective in managing neurological and behavioral symptoms of neuronopathic MPS types I, II and VII, in which pathological changes occur in the central nervous system (CNS), as the enzyme cannot cross the blood-brain barrier (Beck, 2007a, 2007b; Węrrzyn et al., 2010). Another treatment mode is bone marrow transplantation, which has been reported to be efficient only if performed during the first two years of life and only in MPS type I (Beck 2007a, 2007b). Recently, yet another possible therapy was proposed, called gene expression-targeted isoflavone therapy (GET IT), which is a specific kind of substrate reduction therapy
(SRT) and has been suggested to be useful in treatment of various types of mucopolysachcaridoses and other lysosomal storage diseases (Piotrowska et al., 2006; Otomo et al., 2012).

Genistein (5, 7-dihydroxy-3- (4-hydroxyphenyl)-4H1-benzopyran-4-one), a natural isoflavone, significantly inhibits GAG synthesis and decreases GAG storage in fibroblasts of patients suffering from different MPS and mucolipidosis (ML) types (Piotrowska et al., 2006; Otomo et al., 2012). Since genistein has been reported to cross the blood-brain barrier (Tsai, 2005), it was proposed to be useful in the treatment of neurological symptoms of MPS and ML. Studies performed on animal models of MPS II (Friso et al., 2010) and MPS IIIB (Malinowska et al., 2010) showed a significant reduction in lysosomal storage. Clinical studies with MPS III patients performed with the use a genistein-rich isoflavone extract at doses of 5 to $15 \mathrm{mg} / \mathrm{kg} /$ day showed a decrease in GAG levels, improvement of hair morphology, and behavior (Piotrowska et al., 2008, 2011; Delgadillo et al., 2011; Malinova et al., 2012, de Ruijter et al., 2012).

Despite the encouraging results of GET IT and reported clinical benefits for patients (Piotrowska et al., 2008, 2011), some authors suggested a limited or even negligible efficacy of this therapy (Delgadillo et al., 2011). One of the causes of these discrepancies might be unprecise and semi-quantitative biochemical methods of measuring GAG levels. This problem was signalled previously (Piotrowska et al., 2009) and concerns both biological human samples and the material from cell cultures.

One of alternative methods for GAG storage estimation is transmission electron microscopy (TEM) study. In fact, this method has been used for over 45 years to investigate pathology of MPS (Aleu et al., 1965; Lagunoff \& Gritzka, 1966), and TEM analyses were performed recently during tests with different possible medicines (Piotrowska et al., 2006; Jakóbkiewicz-Banecka et al., 2009; Kloska et al., 2011; Dziedzic et al., 2012). The aim of the present work is to assess whether TEM can be used for quantitative estimation of lysosomal storage. To perform such an assessment, TEM analysis was performed in an experimental system in which storage levels could be easily predicted, i.e. in untreated MPS cells and after various treatments.

\footnotetext{
e-mail: magnaraj@biotech.ug.gda.pl
}

Abbreviations: MPS, mucopolysaccharidosis; TEM, transmission electron microscopy; GAG, glycosaminoglycans; ERT, enzyme replacement therapy; SRT, substrat reduction therapy; GET IT, gene expression-targeted isoflavone therapy; CNS, central nervous system; SEM, scaning electron microscopy; ML, mucolipidosis. 


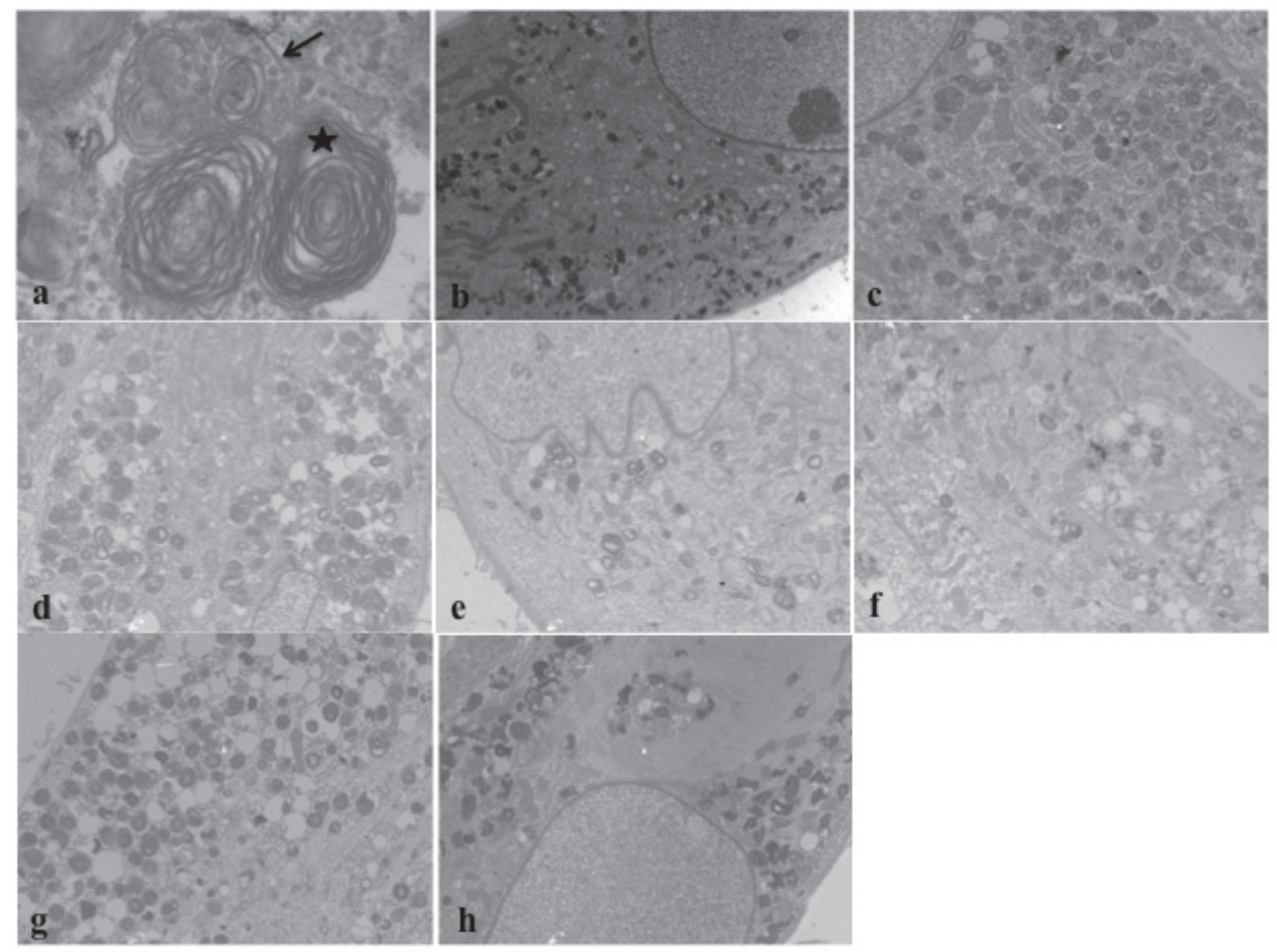

Figure 1. Different lysosomal structures observed in fibroblasts.

Electron micrographs present: (a) lamellar lysosome (asterisk) and complex lysosomal structure (arrow) in the cell, magnification $21000 x$; (b) control (untreated wild type cells); (c) untreated MPS I cells; (d) MPS I cells treated with $30 \mu \mathrm{M}$ genistein; (e) MPS I cells treated with $50 \%$ Aldurazyme and $30 \mu \mathrm{M}$ genistein; (f) MPS I cells treated with 100\% Aldurazyme; (g) untreated MPS IIIA cells; (h) MPS IIIA cells treated with $30 \mu \mathrm{M}$ genistein; all micrographs except panel (a) were made at magnification 1650x.

\section{MATERIALS AND METHODS}

Cell lines and culture conditions. Fibroblast cell lines obtained from MPS I and MPS IIIA patients (Memorial Children Hospital, Warsaw, Poland) were used in experiments. Human Dermal Fibroblast adult line (HDFa; Cascade Biologics, Portland, OR, USA) was used as a healthy control line. Cells were grown in Dulbecco's Modified Eagle Medium (DMEM) supplemented with 10\% Fetal Bovine Serum (FBS) and $1 \times$ Antibiotic and Antimycotic Solution (all purchased from Sigma, Germany) at $37^{\circ} \mathrm{C}$ in humidified $5 \% \mathrm{CO}_{2}$ atmosphere.

Enzyme and genistein. Human recombinant $\alpha-\mathrm{L}-$ iduronidase (Aldurazyme) was obtained from Genzyme Co. (Cambridge, MA, USA). Genistein was purchased from Sigma (Germany).

Electron microscopic studies. Cells were incubated in growth medium supplemented with appropriate concentrations of tested compounds for 14 days. Cells were fixed with $2.5 \%$ glutaraldehyde, followed by PBS washing. Then, the cells were fixed with $1 \%$ osmium tetroxide and 1\% potassium hexacyanoferrate (III) (all purchased from Sigma, Germany), which was followed by ethanol dehydration. Ultrasections (approximately 40 $\mathrm{nm}$ ) of cells embedded in Epon 812 resin (Fluka, Germany) were stained in lead citrate and uranyl acetate (Herman-Antosiewicz et al., 2006), examined under a transmission electron microscope (Philips CM100) and photographed at magnification 1650x using iTEM program (Olympus Soft Imaging Solution). Each experiment was blinded at the time of analysis and reporting. All photographed fibroblasts had intact membrane and visible cells structures (nucleus, mitochondria, lysosomes). At least 20 cells were examined and for each cell at least 5 random fields were assessed. Lamellar lysosomes and complex lysosomal structures (Fig. 1a) were counted per $100 \mu \mathrm{m}^{2}$ of cross-section of a cell.

\section{RESULTS}

The fibroblasts were cultured for 14 days in the absence or presence of different compounds. Following cell preparation, their lysosomal structure was examined under transmission electron microscope (Fig. 1).

Although various abnormal structures were observed, complex lysosomal structures appeared to be the most characteristic for MPS cells. When calculated per 100 $\mu \mathrm{m}^{2}$, the number of these structures correlated well with the expected results of the treatment (the effects of various treatments had been demonstrated previously by other authors and using other methods, see Unger et al., 1994; Piotrowska et al., 2006). Thus, while there were many complex lysosomal structures in untreated 


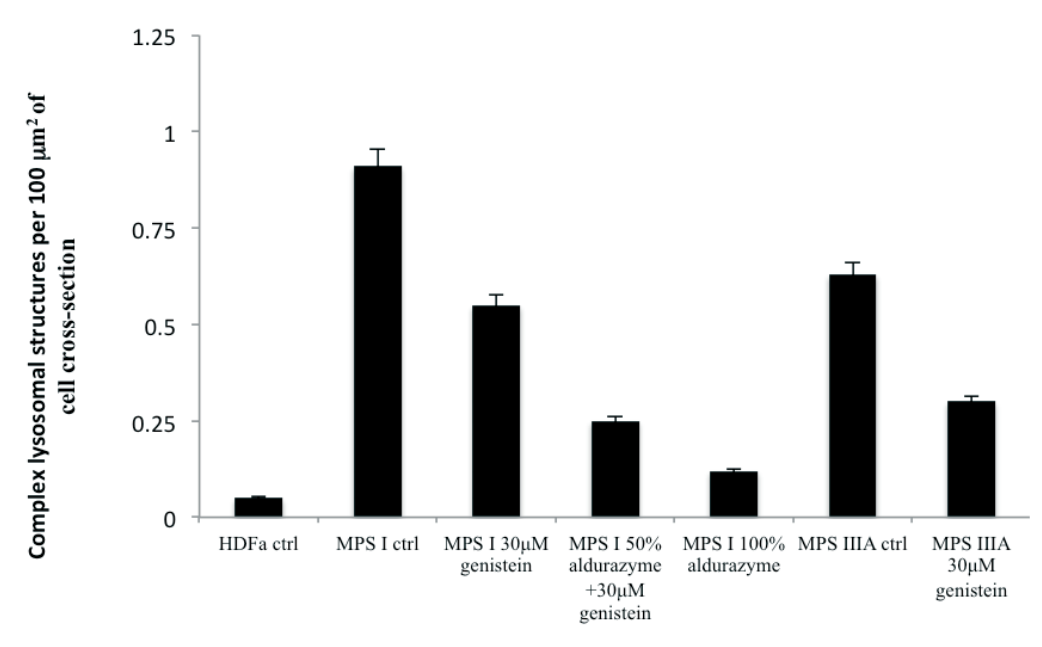

Figure 2. Effects of genistein, Aldurazyme and their combination on the presence of complex lysosomal structures in HDFa, MPS I and MPS IIIA fibroblasts.

The presented values are mean values from 20 analyzed cell (with 5 views of each cell). Error bars indicate S.D.

MPS I and MPS IIIA cells, treatment of MPS I cells with recombinant human $\alpha$-L-iduronidase (Aldurazyme) at the dose recommended for the therapy (described as $100 \%$ dose in this report) resulted in a drastic decrease in this parameter (Fig. 2). Similarly, treatment of MPS I and MPS IIIA cells with $30 \mu \mathrm{M}$ genistein caused a smaller but significant decrease in the number of complex lysosomal structures (Fig. 2). A significant effect was also observed when a combined treatment, 50\% dose of Aldurazyme and $30 \mu \mathrm{M}$ genistein, was applied (Fig. 2). These results strongly suggest that analysis of the frequency of complex lysosomal structures may be an adequate method for estimation of lysosomal storage in MPS.

\section{DISSCUSION}

The use of proper biomarkers in investigation of mechanisms of diseases and in testing therapeutic efficiencies of any medical products is crucial for adequate assessments of the state of the organisms and the disease progress. However, in many diseases it is very difficult to choose an adequate biomarker. Since all types of MPS are causes by accumulation of GAGs (Neufeld $\&$ Muenzer, 2001), determination of levels of these compounds might appear the most straightforward method to assess the disease stage and severity. However, there are various technical problems with precise estimation of this parameter in samples of human tissues or body fluids and cells from in vitro cultures (Piotrowska et al., 2009 and references therein). Most biochemical methods available are only semi-quantitative and are sensitive to the presence of various contaminating compounds in tested samples. Although some sophisticated biochemical methods for GAG analysis have been reported (Korir \& Larive, 2009), they are complicated and difficult to use in standard studies on MPS severity or treatment. Therefore, it was reasonable to look for another method suitable for assessment of lysosomal storage in MPS.

Recently, estimation of hair morphology by scanning electron microscopy (SEM) has been proposed as a possible biomarker in monitoring the disease progress and effects of treatment in MPS (Narajczyk et al., 2012). Although such a method is non-invasive and relatively simple, it can be applied only in clinical studies. In this re- port, a TEM method is proposed for assessment of lysosomal storage in MPS cells. Despite the fact that TEM had been used previously in many studies on MPS, it appeared important to develop a procedure to estimate the level of storage quantitatively.

The results presented in this report suggest that determination of the number of complex lysosomal structures per $100 \mu \mathrm{m}^{2}$ of a cell cross-section may be an adequate measure of pathological state in MPS cells. The results presented in Fig. 2 are compatible with expectations of effects of various treatments of MPS cells on lysosomal storage, based on previously published results where this parameter was carefully investigated by methods (Unger et al., 1994; Piotrowska et al., 2006). Therefore, the use of TEM and determination of the frequency of complex lysosomal structures is suggested as a convenient method for estimation of the level of lysosomal storage in MPS.

\section{Acknowledgements}

This research was supported by the University of Gdańsk (grant no. 538-L107-0806-12) and was operated within the Foundation for Polish Science Team Programme co-financed by the EU European Regional Development Fund (grant no. TEAM/2008-2/7).

\section{REFERENCES}

Aleu FP, Terry RD, Zellweger H (1965) Electron microscopy of two cerebral biopsies in gargoylism. I Neuropathol Exp Neurol 24: 304 317.

Beck M (2007a) Mucopolysaccharidoses: clinical features and management. In Genetic Metabolic Disorders: Management, Costs and Sociomedical Aspects. vom Dahl S, Wendel U, Strohmeyer G, eds, pp 13-18. Cologne: Deutscher Arzte-Verlag.

Beck M (2007b) New therapeutic options for lysosomal storage disorders: enzyme replacement, small molecules and gene therapy. Hum Genet 121: 1-22.

Delgadillo V, O’Callaghan Mdel M, Artuch R, Montero R, Pineda M (2011) Genistein supplementation in patients affected by Sanfilippo disease. J Inherit Metab Dis 34: 1039-1044.

de Ruijter J, Valstar MJ, Narajczyk M, Wegrzyn G, Kulik W, Ijlst L, Wagemans T, van der Wal WM, Wijburg FA (2012) Genistein in Sanfilippo disease: a randomized controlled crossover trial. Ann Neurol 71: 110-120.

Dziedzic D, Narajczyk M, Gabig-Cimińska M, Jakóbkiewicz-Banecka J (2012) Simultaneus siRNA-mediated silencing of pairs of genes coding for enzymes involved in glycosaminoglycan synthesis. Acta Biochim. Pol. 59: 293-298.

Friso A, Tomanin R, Salvalaio M, Scarpa M (2010) Genistein reduces glycosaminoglycan levels in mouse model of mucopolysachcaridosis type II. Br J Pharmacol 159: 1082-1091.

Herman-Antosiewicz A, Johanson DE, Singh SV (2006) Sulfaraphane causes autophagy to inhibit release of cytochrome $\mathrm{c}$ and apoptosis in human prostate cancer cells. Cancer Res 66: 5828-5835.

Jakóbkiewicz-Banecka J, Piotrowska E, Narajczyk M, Barańska S, Węgrzyn G (2009) Genistein-mediated inhibition of glycosaminoglycan synthesis, which correct storage in cells of patients suffering from mucopolysaccharidoses, acts by influencing an epiderma growth factor-dependent pathway. J. Biomem Sci. 16: 26.

Kloska A, Jakóbkiewicz-Banecka J, Narajczyk M, Banecka-Majkutewicz Z, Wegrzyn G (2011) Effects of flavonoids on glycosaminoglycan synthesis: implications for substrate reduction therapy in Sanfilippo disease and other mucopolysaccharidoses. Metab Brain Dis 26: 1-8. 
Korir AK, Larive CK (2009) Advances in the separation, sensitive detection, and characterization of heparin and heparin sulfate. Anal Bioanal Chem 393: 155-169.

Lagunoff D, Gritzka TL (1966) The site of mucopolysaccharide accumulation in Hurler's syndrome. An electron microscopic and histochemical study. Lab Invest 15: 1578-1588.

Malinova V, Wegrzyn G, Narajczyk M (2012) The use of elevated doses of genistein-rich soy extract in the gene expression-targeted isoflavone therapy (GET IT) for Sanfilippo disease patients. IIMD Rep 5: 21-25.

Malinowska M, Wilkinson FL, Bennett W, Langford-Smith KJ, O'Leary HA, Jakobkiewicz-Banecka J, Wynn R, Wraith JE, Wegrzyn G, Bigger BW (2009) Genistein reduces lysosomal storage in peripheral tissues of mucopolysaccharide IIIB mice. Mol Genet Metab 98: 235-242.

Narajczyk M., Tylki-Szymańska A., Węgrzyn G. (2012) Changes in hair morphology as a biomarker in gene expression-targeted isoflavone therapy for Sanfilippo disease. Gene 504: 292-295.

Neufeld EF, Muenzer J (2001) The mucopolysaccharidoses. In The metabolic and molecular bases of inberited disease. Scriver CR, Beaudet AL, Sly WS, Valle D, eds, pp 3421-3452. New York: McGraw-Hill Co.

Otomo T, Hossain MA, Ozono K, Sakai N (2012) Genistein reduces heparin sulfate accumulation in human mucolipidosis II skin fibroblasts. Mol Genet Metabol 105: 266-269.

Piotrowska E, Jakobkiewicz-Banecka J, Baranska S, Tylki-Szymańska A, Czrtoryska B, Węgrzyn A, Węgrzyn G (2006) Genistein-mediated inhibition of glycosaminoglycan synthesis as a basis for gene expression-targeted isoflavone therapy for mucopolysaccharidoses. Eur J Hum Genet 14: 846-852.
Piotrowska E, Jakóbkiewicz-Banecka J, Tylki-Szymańska A, Liberek A, Maryniak A, Malinowska M, Czartoryska B, Puk E, Kloska A, Liberek T, Barańska S, Węgrzyn A, Węgrzyn G (2008) Genistin-rich soy isoflavone extract in substrate reduction therapy for Sanfilippo syndrome: An open-label, pilot study in 10 pediatric patients. Curr Ther Res Clin Exp, 63: 166-179.

Piotrowska E, Jakóbkiewicz-Banecka J, Tylki-Szymańska A, Czartoryska B, Wegrzyn A, Wegrzyn G (2009). Correlation between severity of mucopolysaccharidoses and combination of the residual enzyme activity and efficiency of glycosaminoglycan synthesis. Acta Paediatr 98: 743-749.

Piotrowska E, Jakobkiewicz-Banecka J, Maryniak A, Tylki-Szymanska A, Puk E, Liberek A, Wegrzyn A, Czartoryska B, SlominskaWojewodzka M, Wegrzyn G (2011) Two-year follow-up of Sanfilippo Disease patients treated with a genistein-rich isoflavone extract: assessment of effects on cognitive functions and general status of patients. Med Sci Monit 17: CR196-CR202.

Tsai TH (2005) Concurrent measurement of unbound genistein in the blood, brain and bile of anesthetized rats using microdialysis and its pharmacokinetic application. J Chromatogr A 1073: 317-322.

Unger EG, Durrant J, Anson DS, Hopwood JJ (1994) Recombinant $\alpha$-L-iduronidase: characterization of the purified enzyme and correction of mucopolysaccharidosis type I fibroblasts. Biochem J 304: 43-49.

Wegrzyn G, Jakóbkiewicz-Banecka J, Narajczyk M, Wiśniewski A, Piotrowska E, Gabig-Cimińska M, Kloska A, Słomińska-Wojewódzka M, Korzon-Burakowska A, Wegrzyn A (2010) Why are behaviors of children suffering from various neuronopathic types of mucopolysaccharidoses different? Med Hypoth 75: 605-609. 